\title{
Combinatorial Relations for Digital Pictures
}

\author{
Valentin E. Brimkov ${ }^{1}$, Davide Moroni ${ }^{2}$, and Reneta Barneva ${ }^{3}$ \\ ${ }^{1}$ Mathematics Department, SUNY Buffalo State College, Buffalo, NY 14222, USA \\ brimkove@buffalostate.edu \\ ${ }^{2}$ Laboratory of Signals and Images, Institute of Science and Information \\ Technologies, Area della Ricerca CNR di Pisa, 56124 Pisa, Italy \\ davide.moroni@isti.cnr.it \\ ${ }^{3}$ Department of Computer Science, SUNY Fredonia, NY 14063, USA \\ barneva@cs.fredonia.edu
}

\begin{abstract}
In this paper we define the notion of gap in an arbitrary digital picture $S$ in a digital space of arbitrary dimension. As a main result, we obtain an explicit formula for the number of gaps in $S$ of maximal dimension. We also derive a combinatorial relation for a digital curve.
\end{abstract}

Keywords: Digital geometry, digital picture, gap, brim.

\section{Introduction}

A gap is a location in a digital picture (that is any finite set of pixels/voxels in $2 \mathrm{D} / 3 \mathrm{D})$ through which a "discrete path" can pass. Gaps are considered in rendering pixelized/voxelized scenes, which is done by casting digital rays from the image to the scene [1,2]. Therefore, it is useful to know whether a digital picture has gaps of certain type or is gap-free. This is particularly interesting when dealing with digital curves or surfaces. It is also helpful to have an estimation for the number of gaps (if any) in a considered digital object, possibly as a function of other object parameters. Such kind of information may help better understand the topological structure of a binary picture and is of potential interest in property-based image analysis. Of special interest are the gaps of maximal dimension (to be defined later) since they can be penetrated by a digital ray of any connectivity. Moreover, estimations of the number of such kind of gaps may be useful for evaluating the performance of some polyhedra decomposition algorithms (see comments in Section 4). Moreover, digital picture gap-freeness appears to be equivalent to the notion of well-composedness of a set of pixels proposed by Latecki, Eckhardt, and Rosenfeld [3. This last paper demonstrates the advantages of using well-composed (gap-free) sets in image analysis.

Theoretical studies of this sort are related to combinatorial topology, but are also of interest in several other disciplines, such as digital geometry, combinatorial image analysis, and theory of computer graphics. A classical result is the famous Descartes-Euler formula $v-e+f=2$ that relates the number of vertices $(v)$, edges $(e)$, and facets $(f)$ of a polytope. For various applications of this last formula and other similar results to image analysis and digital geometry, see Chapters 4 and 6 of [4]. 
Conditions for existence of gaps in digital lines and planes are available, e.g., in [5.6 7]. The notion of gap has been used in higher dimensions, too [8]. However, a rigorous definition that applies to arbitrary digital pictures is still missing. Approaches to estimating the number of gaps have been, overall, unclear.

A recent work [9] provided the formula

$$
g=v-2(p+c-h)+b,
$$

where $g$ is the number of gaps, $v$ the number of vertices, $p$ the number of pixels, $h$ the number of holes, $c$ the number of connected components, and $b$ the number of $2 \times 2$ grid squares in a digital picture. For another similar result we refer to $[10$.

In the present paper we define the notion of gap in arbitrary dimension and obtain a formula for the number of gaps of maximal dimension $n$. We also derive a combinatorial relation for an $n$-dimensional digital curve.

In the next section we introduce some basic notions and notations of digital topology. In Section 3 we present our main results. In Section 3.4 we comment on a computer program that was developed to facilitate our theoretical research. We conclude with some remarks in Section 4.

\section{Preliminaries}

In this sections we introduce some basic notions of digital geometry to be used in the sequel. We conform to terminology used in 4] (see also 11]).

All considerations take place in the grid cell model that consists of the grid cells of $\mathbb{Z}^{n}$, together with the related topology. In the grid cell model we represent $n$-cells as hyper-cubes, called hyper-voxels, or voxels, for short. Their edges and vertices are 1 -cells and 0 -cells, respectively. For every $i=0,1, \ldots, n$, the set of all cells of dimension $i$ (or $i$-cells) is denoted by $\mathbb{C}_{n}^{(i)}$. Further, we define the space $\mathbb{C}_{n}=\bigcup_{k=0}^{n} \mathbb{C}_{n}^{(i)}$. We say that two $n$-cells $e, e^{\prime}$ are $k$-adjacent for $0 \leq k \leq n-1$ if they share a $k$-cell. Two $n$-cells are strictly $k$-adjacent if they are $k$-adjacent but not $(k+1)$-adjacent.

A digital object $S \subset \mathbb{C}_{n}$ is a finite set of $n$-cells. A $k$-path $(0 \leq k \leq n-1)$ in $S$ is a sequence of voxels from $S$ such that every two consecutive voxels on the path are $k$-adjacent. Two voxels of a digital object $S$ are $k$-connected (in $S$ ) iff there is a $k$-path in $S$ between them. A subset $G$ of $S$ is $k$-connected iff there is a $k$-path connecting any two pixels of $G$. The maximal (by inclusion) $k$-connected subsets of a digital object $S$ are called $k$-components of $S$. Components are nonempty, and distinct $k$-components are disjoint.

The grid cell model can be considered as an abstract cell complex $\left(\mathbb{C}_{n},<\right.$, dim $)$ (see 12 ), where $<$ is a bounding relation, that is antisymmetric, irreflexive, and transitive, and such that for every $e, e^{\prime} \in \mathbb{C}_{n}, e<e^{\prime}$ if and only if $e I e^{\prime}$ and $\operatorname{dim}(e)<\operatorname{dim}\left(e^{\prime}\right)$. The relation $<$ is a partial order on $\mathbb{C}_{n}$. The corresponding order topology $\tau(<)$ is called the grid cell topology 1 In the rest of the paper,

${ }^{1}$ In that topology the open sets are precisely the sets $U \subseteq \mathbb{C}_{n}$, such that, for every $u \in U$ and every $v \in \mathbb{C}_{n}$ with $u<v$, we have $v \in U$. 
we will assume that the abstract cell complex $\left(\mathbb{C}_{n},<\right.$, dim $)$ is equipped with the topology $\tau(<)$. Then, for any subset $A$ of $\mathbb{C}_{n}$, its boundary $\partial A$ is defined as the set of all points $x$ of $\mathbb{C}_{n}$ such that every open neighborhood of $x$ meets $A$ and $\mathbb{C}_{n} \backslash A$, while its interior $\operatorname{int}(A)$ is the set of all points $x$ of $\mathbb{C}_{n}$ such that there exists some open neighborhood of $x$ contained in $A$. The points of $\operatorname{int}(A)$ will be called internal points of $A$.

Given a digital object $S$, note that its closure $\bar{S}$ is naturally a subcomplex of $\mathbb{C}_{n}$. In the sequel, we will denote by $S_{k}$ the set of $k$-cells of $\bar{S}$, i.e., $S_{k}=\bar{S} \cap \mathbb{C}_{n}^{(k)}$. In particular, we have $S_{n}=\bar{S} \cap C_{n}^{(n)}=S$.

\section{Combinatorial Relations}

In this section we first introduce the notions of tandem, gap, and brim of arbitrary dimension. Then we obtain a formula for the number of gaps of maximal dimension and a combinatorial relation for digital curves.

\subsection{Tandems, Gaps, and Brims}

A $\underbrace{2 \times \cdots \times 2}_{k} \times \underbrace{1 \times \cdots \times 1}_{n-k}$ grid parallelepiped in $\mathbb{C}_{n}$ will be called $2^{k} 1^{n-k}$-block $(0 \leq k \leq n)$. In particular, any voxel is a $1^{n}$-block. See Figure 1 a for illustrations.
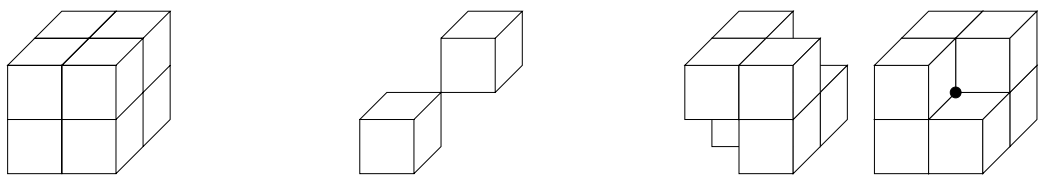

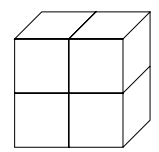

(a)

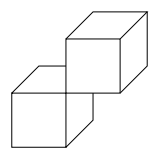

(b)

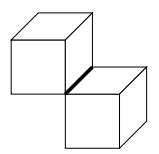

(c)

Fig. 1. Illustration to some notions in 3D. (a) Top: $2^{3}$-block; Bottom: $2^{2} 1^{1}$-block. (b) Top: 0-tandem; Bottom: 1-tandem. (c) Top: Configuration exposing a 0-gap (in two different orientations); Bottom: Configuration exposing a 1-gap.

Now we are able to give the following definition.

Definition 1. A pair $t_{k}=\left(v_{1}, v_{2}\right)$ of two strictly $k$-adjacent voxels $v_{1}$ and $v_{2}$, for $0 \leq k \leq n-1$, is called a $k$-tandem. Then the complement of $t_{k}$ w.r.t. $a$ $2^{n-k} 1^{\bar{k}}$-block, for $0 \leq k \leq n-2$, determines a $k$-gap of $S$. 
Remark 1. Technically, the complement of an $(n-1)$-tandem to a $2^{1} 1^{n-1}$-block can be considered as a $(n-1)$-gap. These are similar to "tunnels" known in classic combinatorial topology, see [4. Since tunnels are well-studied object of essentially diverse type, we will not consider them here.

There are $n-1$ types of gaps: $0,1,2, \ldots$, and $(n-2)$-gaps. For a given digital object $S$, the number of its tandems and gaps will be denoted by $b_{0}, b_{1}, \ldots, b_{n-1}$ and $g_{0}, g_{1}, \ldots, g_{n-2}$, respectively. Figure 1 $1 \mathrm{p}, \mathrm{c}$ illustrates tandems and gaps in dimension three.
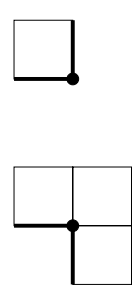
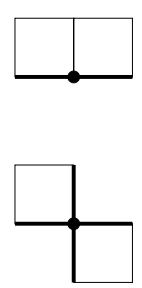
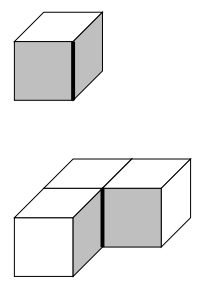
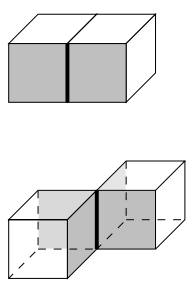

(a)

(b)

In the sequel we will also use the following technical notion.

Definition 2. Let $c \in \partial S_{k-1}$ for some $k(1 \leq k \leq n)$ and let $b_{k}(c)$ be the set of elements of $\partial S_{k}$ incident to it. Then the pair $b_{k}(c)=\left(c, b_{k}(c)\right)$ is called a $k$-brim of $S$. We will say that $b_{k}(c)$ is hinged on $c$.

Basically, $k$-brims of a digital object delineate its " $k$-dimensional" boundary. A set of voxels in a digital object will be called configuration. Figure 2 displays possible configurations of pixels/voxels that expose 1-brims in $\mathbb{C}_{2}$ and 2-brims in $\mathbb{C}_{3}$. (Note that there is one-to-one correspondence between both. There are 19 distinct configurations of voxels that expose 1 -brims in $\mathbb{C}_{3}$.)

\subsection{Formula for the Number of $(n-2)$-Gaps}

For a given digital object $S \subset \mathbb{C}_{n}$, let $s_{i}=\left|S_{i}\right|, 0 \leq k \leq n$. In this section we prove the following theorem.

Theorem 1. For a given digital object $S \subset \mathbb{C}_{n}$,

$$
g_{n-2}=-2 n(n-1) s_{n}+2(n-1) s_{n-1}-s_{n-2}+b,
$$

where $b$ is the number of $2^{2} 1^{n-2}$-blocks of $S$.

Proof. For any $c \in S_{k-1}, 1 \leq k \leq n-1$, we define

$$
I_{k}(c)=\left\{c^{\prime} \in S_{k}: c \text { is incident with } c^{\prime}\right\} .
$$


We also define

$$
\begin{aligned}
\text { int } S_{k-1} & =\left\{c \in S_{k-1}: c \in \text { int } S\right\} \\
\partial S_{k-1} & =\left\{c \in S_{k-1}: c \in \partial S\right\} \\
\partial S_{k} & =\left\{c \in S_{k}: c \in \partial S\right\}
\end{aligned}
$$

It is easy to see that a $(k-1)$-cell belongs to int $S_{k-1}$ iff it is incident with $2^{n-(k-1)} n$-cells of $S$. Otherwise, it belongs to the boundary of $S$.

For $c \in S_{n-1}$ we can consider $I_{n}(c)=\left\{c^{\prime} \in S_{n}: c\right.$ is incident with $\left.c^{\prime}\right\}$. The possible values for $\left|I_{n}(c)\right|$ are 1 and 2 . More precisely, we have

$$
\begin{aligned}
\text { int } S_{n-1} & =\left\{c \in S_{n-1}: I_{n-1}(c)=2\right\} \\
\partial S_{n-1} & =\left\{c \in S_{n-1}: I_{n-1}(c)=1\right\} \\
S_{n-1} & =i n t S_{n-1} \cup \partial S_{n-1}
\end{aligned}
$$

Let us denote $s_{n-1}^{\text {int }}=\mid$ int $S_{n-1} \mid$, and $s_{n-1}^{\partial}=\left|\partial S_{n-1}\right|$. Then $s_{n-1}=s_{n-1}^{\text {int }}+s_{n-1}^{\partial}$. Since every $n$-cell of $S$ is incident with $2 n(n-1)$-cells from $S_{n-1}$, we obtain

$$
2 n|S|=s_{n-1}^{\partial}+2 s_{n-1}^{i n t} .
$$

From here we get

$$
s_{n-1}^{i n t}=n s_{n}-\frac{s_{n-1}^{\partial}}{2} .
$$

Next we consider incidence relations between elements of $\partial S_{n-1}$ and $S_{n-2}$. For any $c \in S_{n-2}$ we consider the brim hinged on $c$ :

$$
b r_{n-1}(c)=\left\{c^{\prime} \in \partial S_{n-1}: c \text { is incident with } c^{\prime}\right\} .
$$

The possible values for $\left|b r_{n-1}(c)\right|$ are 0,2 , and 4 . This partitions $S_{n-2}$ as follows:

$$
S_{n-2}=S_{n-2}^{0} \cup S_{n-2}^{2} \cup S_{n-2}^{4},
$$

where $S_{n-2}^{i}=\left\{c \in S_{n-2}:\left|b r_{n-1}(c)\right|=i\right\}$, for $i=0,2$, 4. If denote $\bar{s}_{n-2}^{i}=$ $\left|S_{n-2}^{i}\right|, i=0,2,4$, we get $s_{n-2}=\bar{s}_{n-2}^{0}+\bar{s}_{n-2}^{2}+\bar{s}_{n-2}^{4}$. From here, we obtain $\bar{s}_{n-2}^{2}=s_{n-2}-\bar{s}_{n-2}^{0}-\bar{s}_{n-2}^{4}$. that

Every cell $x \in S_{n-1}^{\partial}$ is incident with $2(n-1)$ cells $y \in S_{n-2}$. Then it follows

$$
\begin{aligned}
2(n-1) s_{n-1}^{\partial} & =4 \bar{s}_{n-2}^{4}+2 \bar{s}_{n-2}^{2}=4 \bar{s}_{n-2}^{4}+2\left(s_{n-2}-\bar{s}_{n-2}^{0}-\bar{s}_{n-2}^{4}\right)= \\
& =2 \bar{s}_{n-2}^{4}+2 s_{n-2}-2 \bar{s}_{n-2}^{0}
\end{aligned}
$$

from where we obtain

$$
s_{n-1}^{\partial}=\frac{\bar{s}_{n-2}^{4}+s_{n-2}-\bar{s}_{n-2}^{0}}{n-1} .
$$

Then

$$
s_{n-1}=s_{n-1}^{i n t}+s_{n-1}^{\partial}=n s_{n}-\frac{s_{n-1}^{\partial}}{2}+s_{n-1}^{\partial}=n s_{n}+\frac{s_{n-1}^{\partial}}{2}
$$


i.e.,

$$
s_{n-1}=n s_{n}+\frac{\bar{s}_{n-2}^{4}+s_{n-2}-\bar{s}_{n-2}^{0}}{2(n-1)} .
$$

Thus

$$
2(n-1) s_{n-1}=2 n(n-1) s_{n}+\bar{s}_{n-2}^{4}+s_{n-2}-\bar{s}_{n-2}^{0},
$$

and

$$
\bar{s}_{n-2}^{4}=-2 n(n-1) s_{n}+2(n-1) s_{n-1}-s_{n-2}+\bar{s}_{n-2}^{0} .
$$

We also have the following fact.

Fact 1 . For any $n \geq 2$, the sets of $(n-2)$-gaps and $(n-2)$-tandems are determined by the same configurations.

Then it is enough to observe that $\bar{s}_{n-2}^{4}=g_{n-2}$ is the number of $(n-2)$-gaps (that are also $(n-2)$-tandems) and $\bar{s}_{n-2}^{0}=b$ the number of $2^{2} 1^{n-2}$-blocks of $S$, and we obtain the result stated.

Note that for $n=2$ the only gaps in $S$ are the 0 -gaps. For this case equality (4) has the form $s_{1}=2 s_{2}+\frac{1}{2}\left(g_{0}+s_{0}-b\right)$, where $b$ is the number of $(2 \times 2)$-blocks in $S$. Now, by Euler-Poincaré characteristic we have $s_{0}-s_{1}+s_{2}=\beta_{0}-\beta_{1}+\beta_{2}$, where $\beta_{0}, \beta_{1}, \beta_{2}$ are the Betti numbers [4]. From here we get $s_{2}-\left(2 s_{2}+\frac{1}{2}\left(s_{0}-\right.\right.$ $\left.\left.b+g_{0}\right)\right)+s_{0}=\beta_{2}-\beta_{1}+\beta_{0}$.

Since $S$ is homotopic to a $1 \mathrm{D} \mathrm{CW}$-complex, we have $\beta_{2}=0$. Moreover, $\beta_{0}$ is the number of connected components of $S$, while $\beta_{1}$ is the number of its holes. From here we immediately obtain formula (1).

\subsection{Relations for Digital Curves}

A digital curve admits various equivalent definitions [13]. One of them is the following. A simple digital $k$-curve is a set $\Gamma=\left\{c_{1}, c_{2}, \ldots, c_{l}\right\}$ of voxels that satisfy the following two axioms: (A1) $c_{i}$ is $k$-adjacent to $c_{j}$ iff $i=j \pm 1$ (modulo $l$ ), and (A2) $\rho$ is one-dimensional with respect to $k$-adjacency. To get acquainted with the classical definition of dimension of a digital object the reader is referred to [14. For further developments and various results see [13,4] and the bibliography therein. For example, we have the following:

Fact 2. Let $M$ be a finite set of pixels which is one-dimensional with respect to 0 -adjacency. Then $M$ does not contain any $2^{2} 1^{n-2}$-block.

Figure 3 illustrates curves in $\mathbb{C}_{2}$ and $\mathbb{C}_{3}$.

Theorem 2. Let $\Gamma \subset \mathbb{C}_{n}$ be a digital 0-curve. Then:

$$
g_{n-2}=-2 n(n-1) s_{n}+2(n-1) s_{n-1}-s_{n-2} .
$$

Moreover, letting $b_{0}, \ldots b_{n-1}$ be the number of its $k$-tandems, for $0 \leq k \leq n-1$ we have the relation

$$
s_{k}=2^{n-k}\left(\begin{array}{l}
n \\
k
\end{array}\right) s_{n}-\sum_{i=0}^{n-k-1} 2^{i}\left(\begin{array}{c}
k+i \\
k
\end{array}\right) b_{i+k}
$$



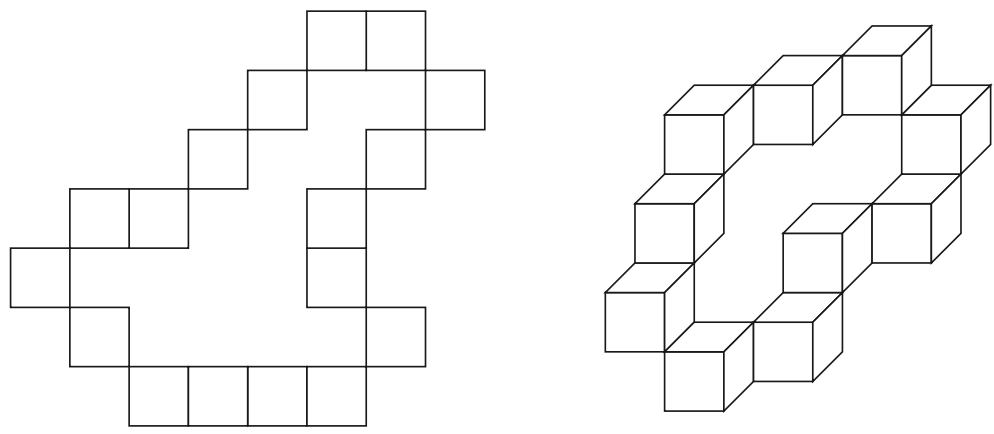

Fig. 3. Simple closed curves in $C_{2}$ (left) and $C_{3}$ (right)

Proof. Let $\Gamma=\left\langle c_{1}, c_{2}, \ldots, c_{m}\right\rangle$ be a closed digital 0-curve, i.e., it satisfies conditions (A1) and (A2) and Fact 2 applies, as well. None that $\Gamma$ consists of consecutive tandems of the form $\left(c_{1}, c_{2}\right),\left(c_{2}, c_{3}\right), \ldots,\left(c_{m-1}, c_{m}\right),\left(c_{m}, c_{1}\right)$.

The first assertion follows immediately from Theorem 1 and Fact 2 .

For the second assertion, let $c$ be a $k$-cell for $k \neq n$.

We say that $c$ is a totally boundary cell if $c$ is incident with exactly one $n$-cell. If $c$ is not totally boundary, then $c$ belongs to the closure of the shared face of a tandem $t_{j}$ in dimension $j \geq k$; we then say that $c$ is involved in $t_{j}$.

Since $\Gamma$ is a 0 -curve, every $k$-cell is incident with at most two $n$-cells and, thus, every non totally boundary cell is involved in exactly one tandem. Now the number of $k$-cells involved in a $j$-dimensional tandem $t_{j}$ is easily seen to be $2^{j-k}\left(\begin{array}{l}j \\ k\end{array}\right)$. Therefore the number of non totally boundary cells $s_{k}^{\text {ntb }}$ is:

$$
s_{k}^{\mathrm{ntb}}=b_{k}+2\left(\begin{array}{c}
k+1 \\
k
\end{array}\right) b_{k+1}+\ldots+2^{n-1-k}\left(\begin{array}{c}
n-1 \\
k
\end{array}\right) b_{n-1},
$$

whereas the number of totally boundary $k$-cells is given by $s_{k}^{\text {tb }}=s_{k}-s_{k}^{\text {ntb }}$. Since every $n$-cell is incident with $2^{n-k}\left(\begin{array}{l}n \\ k\end{array}\right) \mathrm{k}$-cells, we have:

$$
\begin{aligned}
2^{n-k}\left(\begin{array}{l}
n \\
k
\end{array}\right) s_{n} & =1 \cdot s_{k}^{\mathrm{tb}}+2 \cdot s_{k}^{\mathrm{ntb}} \\
& =s_{k}+s_{k}^{\mathrm{ntb}}
\end{aligned}
$$

The second assertion now follows straightforwardly from eq. (6) and eq. (77).

Remark 2. Note that $(n-2)$-gaps are the only gaps a digital curve $\Gamma$ may have. Note also that if $\Gamma$ is a digital $(n-2)$-curve 2 then the number of $(n-2)$-gaps of $\Gamma$ matches the number of "linear segments" into which $\Gamma$ can be decomposed.

Remark 3. Since $\Gamma$ is a closed curve, its Euler-Poincaré characteristic $\chi(\Gamma)$ is zero. We then have:

$$
0=\chi(\Gamma)=\sum_{k=0}^{n}(-1)^{k} s_{k}
$$

\footnotetext{
${ }^{2}$ That is, any two consecutive voxels of $\Gamma$ are $(n-2)$-adjacent.
} 


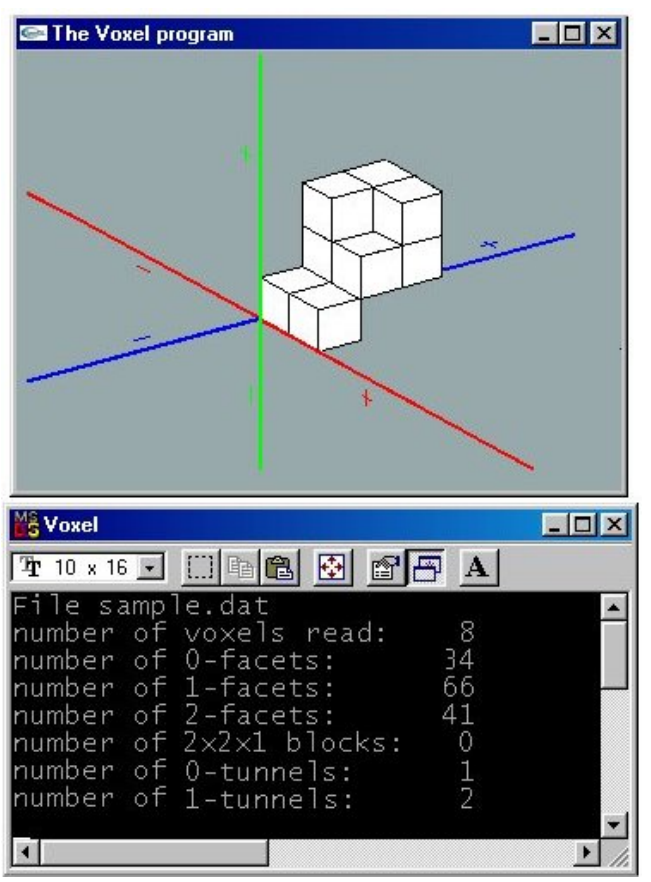

Fig. 4. Sample output of the computer program

Using the expression for the $s_{i}$ found in eq. (5), we recover, after elementary manipulations, the not-surprising relation:

$$
s_{n}=b_{0}+b_{1}+\ldots+b_{n-1}
$$

The used approach allows to obtain similar (although more complex and thus less compact and elegant) relations for $k$-curves with $k \neq 0$, as well as for arbitrary digital object.

\subsection{Experimental Software}

The theoretical results described in the previous sections have been supported and verified by an experimental computer program.

Given a digital picture $S$ represented by the coordinates of its voxels, our program takes as an input a file with the list of the voxel coordinates. It outputs the number of the 0 -, 1 -, and 2 -facets, $2^{2} 1^{1}$-blocks, and 0 - and 1 -gaps of $S$. Computation of the number of the 0-/1-gaps is performed by appropriate scanning of $S$ by $2 \times 2 \times 2$-cubes $/ 2 \times 2 \times 1$ - blocks and counting the distinct gaps. The number of 1-gaps can alternatively be found by using formula (2).

The program is written in Visual Studio $\mathrm{C}++6.0$ and uses OpenGL. It runs under Windows 98 or higher. It allows to visualize the digital picture $S$ and to 
interactively rotate it along the $\mathrm{Ox}_{-}, \mathrm{Oy}$-, and $\mathrm{Oz}$ - axes so that the object can be seen from different viewpoints. In Figure 4 a snapshot of the running program is displayed.

\section{Concluding Remarks}

In this paper we provided a rigorous definition of gaps in a digital picture and derived a formula for the number of $(n-2)$-gaps, as well as certain combinatorial relations for digital curves. A supporting computer program has been developed as well.

Knowledge of the number of gaps of maximal dimension can be useful in several aspects. Among these we would like to mention an application to the wellknown polyhedron decomposition problem [15, 16, that is to partition a given non-convex polyhedron into as small as possible number of convex polytopes. Specifically, let $P$ be the rectilinear polyhedron defined as a union of a set of voxels of $\mathbb{C}_{3}$. It is not hard to see that the number of gaps in the discrete surface constituted by the boundary voxels of $P$ is an upper bound for the number $r$ of "notches" of $P$, that are locations causing non-convexity 3 The fact is that all bounds on the number of convex polytopes obtained by decomposition algorithms are in terms of that parameter $r$. A more careful study of this aspect is seen as a further task. Another one is seen in seeking approaches that would allow to obtain more compact characterizations of lower dimensional gaps in digital pictures.

\section{Acknowledgements}

The authors thank the two anonymous referees for a number of useful remarks and suggestions.

\section{References}

1. Cohen-Or, D., Kaufman, A.: 3D Line Voxelization and Connectivity Control. IEEE Computer Graphics and Applications 17 (6) (1997) 80-87

2. Kaufman, A., Cohen, D., Yagel, R.: Volume Graphics. IEEE Computer 26 (7) (1993) 51-64

3. Latecki, L., Eckhardt, U., Rosenfeld, A.: Well-Composed Sets. Computer Vision and Vision Understanding 61 (1995) 70-83

4. Klette, R., Rosenfeld, A.: Digital Geometry - Geometric Methods for Digital Picture Analysis. Morgan Kaufmann, San Francisco (2004)

5. Brimkov, V.E., Coeurjolly, D., Klette, R.: Digital Planarity - a Review. CITR-TR 142, Auckland (2004)

${ }^{3}$ Notch (or reflex edge) is an edge of a polyhedron where the inner dihedral angle subtended by two incident facets is greater than 180 degrees. 
6. Andres, E., Nehlig, Ph., Françon, J.: Tunnel-Free Supercover 3D Polygons and Polyhedra. In: Fellner D., Szirmay-Kalos L. (guest eds.): EUROGRAPHICS'97 (1997) C3-C13

7. Brimkov, V.E., Andres, E., Barneva, R.P.: Object Discretizations in Higher Dimensions. Pattern Recognition Letters 23 (2002) 623-636

8. Andres, E., Acharya, R., Sibata, C.: Discrete Analytical Hyperplanes. Graphical Models and Image Processing 59 (1997) 302-309

9. Brimkov, V.E., Maimone, A., Nordo, G., Barneva, R.P., Klette, R.: The Number of Gaps in Binary Pictures. In: Bebis et al. (eds.): Advances in Visual Computing. Lecture Notes in Computer Science, Vol. 3804. Springer-Verlag, Berlin Heidelberg New York (2005) 35-42

10. Brimkov, V.E., Maimone, A., Nordo, G.: Counting Gaps in Binary Pictures. In: 11th International Workshop on Combinatorial Image Analysis. Lecture Notes in Computer Science, Vol. 4040. Springer-Verlag, Berlin Heidelberg New York (2006) $16-24$

11. Kong, T.Y.: Digital Topology. In: Davis, L.S. (ed.): Foundations of Image Understanding. Kluwer, Boston Massachusetts (2001) 33-71

12. Kovalevsky, V.A.: Finite Topology as Applied to Image analysis. Computer Vision, Graphics and Image Processing 46(2) (1989) 141-161

13. Brimkov, V.E., Klette, R.: Curves, Hypersurfaces, and Good Pairs of Adjacency Relations. In: Klette, R., Zunic, J.K. (eds.): 10th International Workshop on Combinatorial Image Analysis. Lecture Notes in Computer Science, Vol. 3322. SpringerVerlag, Berlin Heidelberg New York (2004) 270-284

14. Mylopoulos, J.P., Pavlidis, T.: On the Topological Properties of Quantized Spaces. I. The Notion of Dimension. J. ACM 18 (1971) 239-246

15. Dielissen, V.J., Kaldewaij, A.: Rectangular Partition is Polynomial in Two Dimensions but NP-complete in Three. Information Processing Letters 38 (1991) 1-6

16. Hershberger, J.E., Snoeyink, J.S.: Erased Arrangements of Lines and Convex Decompositions of Polyhedra. Computational Geometry: Theory and Applications 9 (1998) 129-143 